\title{
A Bout of Vertigo, Subsequent Diarrhea and Nausea Highlighting a Spectrum of Possible Ailments
}

\author{
Daniel Benharroch, M.D* \\ Department of Pathology, Soroka University Medical Center, and Faculty of Health Sciences, Ben-Gurion \\ University of the Negev, Beer-Sheva, Israel
}

*Corresponding Author: Prof. Daniel Benharroch, Department of Pathology, Soroka University Medical Centre, Rager Blvd, P.O.Box 151, Beer-Sheva 84101, Israel.

\begin{abstract}
A 71 year-old man was driving his car by bright day time, when he was overwhelmed by a sudden and robust fit of vertigo. With great difficulties, he succeeded in parking the car by the road edge. The bout of vertigo abated gradually, leaving behind residual nausea. A few hours later, the patient developed diarrhea, with marked colicky abdominal pain. Diarrhea and nausea persisted for several additional hours. The report of this incident concerns the differential diagnosis of this medical episode. Of note, the spell did not last more than a few hours.
\end{abstract}

Running Title: Acute vertigo, diarrhea, and the differential diagnosis.

Keywords: vertigo; diarrhea; spell; colicky pain; vertebro-basilar; food poisoning; COVID-19

\section{INTRODUCTION}

An elderly individual, who endures a severe bout of vertigo, might be categorized as a cerebrovascular accident-suspect until proven otherwise. Likewise, an ailment originating in the internal ear may also be suggested.

However, when vertigo is compounded with diarrhea and nausea, an infectious disease should be contemplated [1]. Moreover, one might also bear in mind a possible poisoning, as the cause of the above symptoms [2]. In addition, an isolated report has also included COVID-19 in association with the given symptoms [3].

\section{Presentation of the Case}

The story of a 71 year-old man, who was caught off-guard by a severe, acute, attack of vertigo, while driving in full day light, is unfolded. He felt the landscape whirling about his car. He was lucky enough to keep the control of the car wheel and to bring the vehicle to a complete arrest. The spell lasted a further $15 \mathrm{~min}$, and then receded progressively. He drove a further $1.5 \mathrm{~km}$ to reach home. The subject remained dizzy for a further $2 \mathrm{~h}$, after which he started to move around, complaining of nausea. By then, an abdominal colicky pain had developed, and diarrhea followed, that progressively changed into soft stools for another $35 \mathrm{~h}$.

The patient had noted a marked prior rhinorrhea lasting for more than a month, prominent mainly in the morning. Neither headache, nor persistent dizziness had marked this period. A diagnosis of sinusitis had never been established with any degree of certainty.

The old man recalled having eaten a tuna sandwich, several hours before the above reported incident. Relatively frequent accounts of food poisoning, following ingestion of tuna fish have been lately described in Israel, but none referred to mercury intoxication. In the absence of his personal GP, and since his condition had improved, the patient did not consult. He was left with remains of rhinorrhea and soft stools.

No history of exposure to lead, and no mercury poisoning have been evoked. No ingestion of exotic fish was reported. Since no evidence of symptomatic COVID-19 was identified, and as a single case only of vertigo associated with diarrhea, confirmed as COVID-19 has been published [3], no RT-PCR for SARS-CoV-2 was performed. Moreover, these symptoms have not been classified as necessary or sufficient to 
require the performance of molecular confirmation of the infectious disease.

\section{DISCUSSION}

Of the several conditions, the diagnosis of which has been raised for this patient, some present a higher level of probability.

In spite of the short span of the given episode, a vestibular-derived disorder should be excluded at an early stage. But the lack of perseverance of vertigo, is not contributory to establish this category of condition, especially not in a context of diarrhea. Acute and chronic sinusitis should probably be excluded.

The specific add up of diarrhea increases the chances that we are dealing with an infectious disease, which might be boosted by the tuna sandwich, guiding us to favor food poisoning [1]. And indeed, food poisoning in the context of tuna fish ingestion represents a much more frequent occurrence than mercury poisoning in Israel $[4,5]$. Lead poisoning, perhaps presenting similarly, must display specific significant antecedents [6, abstract only, 7].

Vertigo, presenting with diarrhea has occurred in severe instances of cerebrovascular accidents or even of acute myocardial infarction, imposing an overwhelming toll on the victims.

Last, a further differential relates with a rare occurrence, described in a single report. In this episode, the subject complained of severe vertigo. He later developed diarrhea and has been eventually confirmed as a COVID-19 patient [3].

\section{Limitations AND CONCLUSIONS}

The differential diagnosis associated with the two main symptoms, vertigo and diarrhea, might be limited. The most probable diagnosis, in my view, is food poisoning. The unusual nature of the medical history, concerns the blast-like episode of vertigo that is highlighted in this patient.

Regarding the possibility of COVID-19, a RTPCR, should have been performed. However, the odds are most probably against the occurrence of this infectious disease.

\section{ACKNOWLEDGEMENTS}

We thank the Department for the thorough discussion.

The authors declare 'No conflict of evidence exists'.

No funding was necessary.

\section{REFERENCES}

[1] Singh M, Kalghatgi AT, Narayanan K, et al. Outbreak of Salmonella food poisoning at high altitude. Med J Armed Forces India. 1998;54:9698.

[2] Bagnis R, Kuberski T, Laugier S. Clinical observations on 3,009 cases of ciguatera (fish poisoning) in the South Pacific. Am J Trop Med Hyg. 1979;28:1067-73.

[3] Liu C, Zhou J, Xia L, et al. 18FF-FDG PET/CT and serial chest CT findings in a COVID-19 patient with dynamic clinical characteristics in different periods. ClinNucl Med. 2020.

[4] Hirschhorn N, Greaves IA. Louisa May Alcott: her mysterious illness. PerspectBiol Med. 2007;50:243-59.

[5] de Vocht F, van Drooge H, Engels H, Kroumhout H. Exposure, health complaints and cognitive performance among employees of an MRI scanner manufacturing department. J MagnReson Imaging. 2006;23:197-204.

[6] Karai I, Nishikawa N, Horigushi S. [A case of optic atrophy of a lead worker]. Sangyo Igaku. 1981; 23:227-32 (Abstract only).

[7] Wilson AT. Acute vertigo and the lead content of food and drink. Practitioner. 1968;200:282-5.

Citation: Daniel Benharroch, M.D. A Bout of Vertigo, Subsequent Diarrhea and Nausea Highlighting a Spectrum of Possible Ailments. ARC Journal of Clinical Case Reports. 2020; 6(3):13-14. DOI:https://doi.org/10.20431/2455-9806.0603003.

Copyright: () 2020 Authors. This is an open-access article distributed under the terms of the Creative Commons Attribution License, which permits unrestricted use, distribution, and reproduction in any medium, provided the original author and source are credited. 